\title{
Crystal chemistry of defects in copper- and oxygen-doped zinc chalcogenide crystals
}

\author{
Dmytro FREIK ${ }^{1 *}$, Nazar STASHKO ${ }^{1}$, Nataliya FREIK ${ }^{1}$, Lyubov MEZHYLOVSKA ${ }^{1}$, Galyna GURGULA ${ }^{1}$ \\ ${ }^{1}$ Department of Solid State Physics and Chemistry, Precarpathian Vasyl Stefanyk University, \\ Shevchenka St. 57, 76000 Ivano-Frankivsk, Ukraine \\ * Corresponding author.Tel.: +380-342503752; fax: +380-342503752; e-mail: fcss@pu.if.ua
}

Received July 8, 2009; accepted December 23, 2009; available on-line April 27, 2010

Generalized quasi-chemical formula for $\mathrm{Zn} X$ crystals $(X=\mathrm{S}$, Se) with $\mathrm{O}$ and $\mathrm{Cu}$ admixtures, taking into account both intrinsic point defects and complexes formed with the doping elements, are proposed. The dependence of the concentration of defects and the Hall concentration of the current carriers on the content of the $\mathrm{Cu}$ doping agent was calculated for different values of residual oxygen concentration both for stoichiometric zinc chalcogenides and for $\mathrm{n}$ - and $\mathrm{p}-\mathrm{ZnX}$. The role of copper and oxygen in the formation of the electronic subsystem of the $\mathrm{Zn} X$ crystals and in the realization of thermodynamic p-n transitions was investigated. It is shown that if $[\mathrm{O}]>[\mathrm{Cu}]$, for crystals with n-type conductivity $V_{Z n}^{\prime \prime}, V_{X}^{\bullet}$, and $Z n_{i}^{\bullet}$ point defects and their complexes $\left(O_{X}^{\times} Z n_{i}^{\bullet} V_{Z n}^{\prime \prime}\right)^{\prime},\left(O_{X}^{\times} C u_{i}^{\bullet} V_{Z n}^{\prime \prime}\right)^{\prime}$ prevail, whereas for p-type crystals, $V_{Z n}^{\prime \prime},\left(O_{X}^{\times} Z n_{i}^{\bullet \bullet} V_{Z n}^{\prime}\right)^{\bullet}$, and $\left(O_{X}^{\times} C u_{i}^{\bullet \bullet} V_{Z n}^{\prime}\right)^{\bullet}$ dominate. If $[\mathbf{C u}]>[O]$, for $\mathbf{n}-Z \mathbf{n X} V_{Z n}^{\prime \prime}, V_{X}^{\bullet}, Z n_{i}^{\bullet}$ point defects and $\left(O_{X}^{\times} C u_{i}^{\bullet} V_{Z n}^{\prime \prime}\right)^{\prime}$ complex prevail, while for p-type crystals $V_{Z n}^{\prime \prime}, V_{S e}^{\bullet}$ and $\left(O_{X}^{\times} C u_{i}^{\bullet \cdot} V_{Z n}^{\prime}\right)^{\bullet}$ are found.

Crystals / Zinc chalcogenides / Oxygen complexes / Quasi-chemical formulae / Point defects

\section{Кристалохімія дефектів у легованих міддю і киснем кристалах цинк халькогенідів}

\author{
Дмитро ФРЕЇК ${ }^{1 *}$, Назар СТАШКО ${ }^{1}$, Наталія ФРЕЇК ${ }^{1}$, Любов МЕЖИЛОВСЬКА ${ }^{1}$, Галина ГУРГУЛА ${ }^{1}$ \\ ${ }^{1}$ Кафедра фізики і хімії твердого тіла, Прикарпатський національний університет імені Василя \\ Стефаника, вул. Шевченка 57, 76000 Івано-Франківськ, Украӥна \\ * Контактна особа. Тел.: +380-342503752; факс: +380-342503752; e-mail: fcss@ pu.if.ua
}

Вперше запропоновано узагальнені кристалоквазіхімічні формули для кристалів $\mathrm{Zn} X(X=\mathrm{S}, \mathrm{Se})$ iз домішками О та Сu, які враховують як власні точкові дефекти, так i комплекси із легуючими елементами. Розраховано залежності концентрації дефектів, холлівської концентрації носіїв струму від вмісту легуючої домішки Сu для різних значень концентрації фонового кисню, як у стехіометричних цинк халькогенідах, так i n- та p-ZnX. Визначено роль кисню і міді у формуванні електронної підсистеми кристалів $\mathrm{Zn} X$ i реалізації у них термодинамічних p-n переходів. Показано, що за умови [O] > [Cu] якщо для кристалів n-типу провідності переважають точкові дефекти $V_{Z n}^{\prime \prime}, V_{X}^{\bullet}$, $Z n_{i}^{\bullet}$ та їх комплекси $\left(o_{X}^{\times} Z n_{i}^{\bullet} V_{Z n}^{\prime \prime}\right)^{\prime},\left(O_{X}^{\times} C u_{i}^{\bullet} V_{Z n}^{\prime \prime}\right)^{\prime}$, то для р-типу $-V_{Z n}^{\prime \prime}$ та $\left(O_{X}^{\times} Z n_{i}^{\bullet \bullet} V_{Z n}^{\prime}\right)^{\bullet},\left(O_{X}^{\times} C u_{i}^{\bullet \bullet} V_{Z n}^{\prime}\right)^{\bullet}$. При [Cu] $>$ [O] для $\mathbf{n - ~} Z \mathbf{n X}$ домінують $V_{Z n}^{\prime \prime}, V_{X}^{\bullet}, Z n_{i}^{\bullet}$ та комплекс $\left(o_{X}^{\times} C u_{i}^{\bullet} V_{Z n}^{\prime \prime}\right)^{\prime}$, а для р-типу - $V_{Z n}^{\prime \prime}, V_{S e}^{\bullet} \mathbf{i}$ $\left(O_{X}^{\times} C u_{i}^{\bullet \bullet} V_{Z n}^{\prime}\right)^{\bullet}$.

Кристали / Цинк халькогеніди / Кисневі комплекси / Кристалоквазіхімічні формули / Точкові дефекти 


\section{Вступ}

Цинк халькогеніди - перспективні матеріали оптоелектроніки, оскільки їхній робочий діапазон відповідає синьо-блакитній області оптичного спектру [1]. Встановлено, що спектральний склад смуг випромінювання $\mathrm{Zn} X$ суттєво залежить від легуючих домішок. Найбільш поширеною фоновою домішкою у напівпровідникових матеріалах $є$ кисень [1,2]. При наявності фонової домішки кисню у кристалах $A^{\mathrm{II}} B^{\mathrm{VI}}$ характерними $\epsilon$ три різні смуги катодолюмінесценції, які ідентифіковані в літературі як SA(I), SAL(II) i III [3]. Додаткове легування міддю пов'язують із центрами $\mathrm{Cu}(\mathrm{I}), \quad \mathrm{Cu}(\mathrm{II})$ i $\mathrm{Cu}(\mathrm{III})$ відповідно (Таблиця 1) [3]. Цим смугам відповідають комплекси із атомами кисню $O_{X}^{\times}$, які знаходяться у вузлах кристалічної гратки та утворюють ізоелектронні центри. Вони зумовлюють зменшення відстаней між взаємодіючими частками кристалічної гратки до 40-50 \%, що у першому наближенні можна представити як утворення нових власних точкових дефектів міжвузлового цинку $Z n_{\mathrm{i}}$ та вакансії цинку $V_{\mathrm{Zn}}$ (Рис. 1).

Зарядовий стан відзначених центрів катодолюмінесценції визначається типом, концентрацією i зарядом власних точкових дефектів, які пов'язані із відхиленнями від стехіометричного складу основної матриці (Рис. 2). Так, зокрема, смуги $\mathrm{SA}(\mathrm{I})$ i $\mathrm{Cu}(\mathrm{I})$, за які відповідають комплекси відповідно $\left(O_{X}^{\times} Z n_{i}^{\bullet} V_{Z n}^{\prime \prime}\right)^{\prime}$

та $\left(O_{X}^{\times} C u_{i}^{\bullet} V_{Z n}^{\prime \prime}\right)^{\prime}$ характерні для кристалів, збагачених цинком (Таблиця 1). При цьому центр $\mathrm{SA}(\mathrm{I})$ - смуга катодолюмінесценції з максимумом $\sim 630$ нм, що пов'язана 3 активацією киснем. Відповідальний за це випромінювання комплекс включає кисень у вузлі гратки, вакансію цинку $V_{Z n}^{\prime \prime}$ і $Z n_{i}^{\bullet}$ (Рис. 1а). При цьому сумісно із цією смугою виникає смуга $\mathrm{Cu}(\mathrm{I})$ - 640 нм, що пов'язана із мідним центром (Рис. 2a). Ці смуги досить близькі і тому їх важко ідентифікувати. Смуги $\mathrm{SAL}(\mathrm{II})$ i $\mathrm{Cu}(\mathrm{II})$, яким відповідають комплекси $\left(O_{X}^{\times} Z n_{i}^{\bullet \bullet} V_{Z n}^{\prime \prime}\right)^{\times}$та $\left(O_{X}^{\times} C u_{i}^{\bullet \bullet} V_{Z n}^{\prime \prime}\right)^{x}$ (Таблиця 1), формуються за технологічних факторів, що забезпечують склад, близький до стехіометричного, або ж незначне збагачення халькогеном. Смуги III (735 нм), Cu(III) (740 нм) виникають у $\mathrm{Zn} X$ за умови значного надлишку халькогену (Рис. 2б), який зумовлює пониження рівня Фермі і збільшення концентарції власних однозарядних акцепторів $V_{Z n}^{\prime}$, що відповідають комплексам $\left(O_{X}^{\times} Z n_{i}^{\bullet \bullet} V_{Z n}^{\prime}\right)^{\bullet}$ або $\left(O_{X}^{\times} C u_{i}^{\bullet \bullet} V_{Z n}^{\prime}\right)^{\bullet}$ (Рис. 1б) відповідно для нелегованих і легованих міддю кристалів.
Зауважимо, що у літературі до цього часу не встановлені кількісні співвідношення між різними типами дефектів i вплив на них легуючих домішок, не визначено залежність фізико-хімічних властивостей, зокрема, типу провідності від концентрації легуючих елементів.

У даній роботі на основі кристалоквазіхімічних формул для чистих i легованих кристалів цинк халькогенідів зроблено аналіз впливу кисню та міді на механізми формування дефектної підсистеми.

\section{Нестехіометричні кристали}

Кристалоквазіхімічний метод опису дефектної підсистеми кристалів згідно 3 роботою [4] полягає у суперпозиції кристалохімічних кластерів базової i легуючої сполук, утворених на основі антиструктури основної матриці. Для цинк халькогенідів $\mathrm{Zn} X$ антиструктурою $\epsilon$ сфалерит $V_{Z n}^{\prime \prime} V_{S e}^{\bullet . ~ Т у т ~} V_{Z n}^{\prime \prime}$ і $V_{S e}^{\bullet \bullet}$ - вакансії Zn і $X$, “/” і “॰” негативний і позитивний заряди відповідно.

Легуючий кластер для $\mathrm{n}-\mathrm{Zn} X$ (надлишок цинку у межах області гомогенності) за умови часткового $(\gamma)$ заміщення цинком вакансій у катіонній підгратці $\left(Z n_{Z n}^{\prime \prime}\right)$ і утворення міжвузлового цинку $\left(Z n_{i}^{\times}\right)$можна представити як: $V_{Z n}^{\prime \prime} V_{\ddot{X}}+Z n^{0} \rightarrow\left(Z n_{\gamma}^{\prime \prime} V_{(1-\gamma)}^{\prime \prime}\right)_{Z n} V_{\ddot{X}}\left(Z n_{(1-\gamma)}^{\times}\right)_{i}$, а кристалоквазіхімічну формулу:

$$
\begin{aligned}
& (1-\alpha) Z n_{Z n}^{\times} X_{X}^{\times}+\alpha\left(Z n_{\gamma}^{\prime \prime} V_{(1-\gamma)}^{\prime \prime}\right)_{Z n} V_{X}^{\cdot .}\left(Z n_{(1-\gamma)}^{\times}\right)_{i} \rightarrow \\
& \left(Z n_{(1-\alpha)}^{\times} Z n_{\alpha \gamma}^{\prime \prime} V_{\alpha(1-\gamma)}^{\prime \prime}\right)_{Z n}\left(X_{1-\alpha}^{\times} V_{\alpha}^{. \prime}\right)_{X}\left(Z n_{\alpha(1-\gamma)}^{\times}\right)_{i} \rightarrow \\
& \left(Z n_{(1-\alpha)+\alpha \gamma}^{\times} V_{\alpha(1-\gamma)}^{\prime \prime}\right)_{Z n}\left(X_{1-\alpha}^{\times} V_{\alpha}^{. \prime}\right)_{X} \times \\
& \left(Z n_{\alpha(1-\gamma)(1-\delta)}^{\cdot} n_{\alpha(1-\gamma) \delta}\right)_{i}+\alpha(1+\gamma+\delta-\gamma \delta) e^{\prime}
\end{aligned}
$$

де $\alpha$ - атомна частка легуючого кластера. Зауважимо, що в (1) враховано

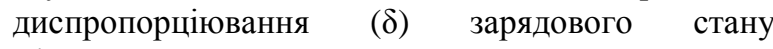
міжвузлового цинку $\left(Z n_{\alpha(1-\gamma)}^{\times}\right)_{i} \rightarrow\left(Z n_{\alpha(1-\gamma)(1-\delta)} Z n_{\alpha(1-\gamma) \delta}\right)_{i}+\alpha(1+\delta-\gamma-\gamma \delta) e^{\prime}$

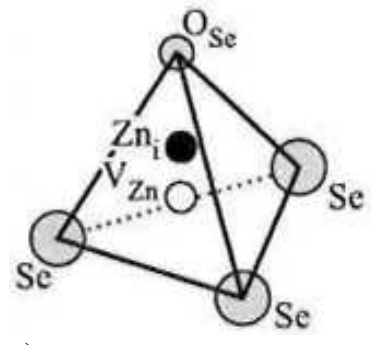

a)

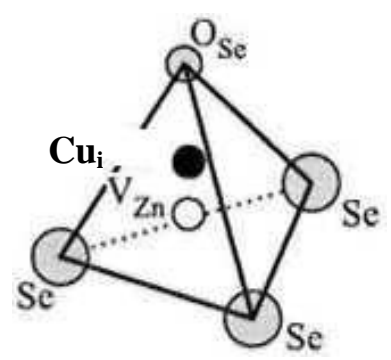

б)
Рис.1 Елемент структури гратки сфалериту кристалів $\mathrm{ZnSe:} \mathrm{тетраедричне} \mathrm{оточення} \mathrm{катіонного}$ вузла для комплексів 3 цинком (а) та міддю (б). 
D. Freik et al., Crystal chemistry of defects in copper- and oxygen-doped zinc chalcogenide crystals

Таблиця 1 Співставлення смуг випромінювання самоактивованих кисневих і мідних центрів в ZnX.

\begin{tabular}{|c|c|c|c|}
\hline \multirow{2}{*}{ Характеристика центра } & \multirow{2}{*}{ Зарядовий стан } & \multicolumn{2}{|c|}{ Смуги випромінювання: $\lambda$, нм; $\mathrm{E}_{\mathrm{A}}, \mathrm{eB}$} \\
\hline & & $\mathrm{ZnSe}$ & $\mathrm{ZnS}$ \\
\hline $\begin{array}{c}\mathrm{SA}(\mathrm{I}) \\
\left(O_{X}^{\times} Z n_{i}^{*} V_{Z n}^{\prime \prime}\right)^{\prime} \\
\text { Надлишок Цинку } \\
\mathrm{Cu}(\mathrm{I}) \\
\left(O_{X}^{\times} \mathrm{Cu} \cdot V_{i}^{*} V_{Z n}^{\prime \prime}\right)^{\prime} \\
\end{array}$ & $I^{\prime}$ & $\begin{array}{c}\lambda=628(605) \\
\mathrm{E}_{\mathrm{A}}=0.61(0.55) \\
\lambda=640 \\
\mathrm{E}_{\mathrm{A}}=0.65\end{array}$ & $\begin{array}{c}\lambda=445(415) \\
\mathrm{E}_{\mathrm{A}}=0.69(0.59) \\
\lambda=450 \\
\mathrm{E}_{\mathrm{A}}=0.7\end{array}$ \\
\hline $\begin{array}{c}\mathrm{SA}(\mathrm{II}) \\
\left(o_{X}^{\times} Z n_{i}^{\cdot \cdot} V_{Z n}^{\prime \prime}\right)^{\times} \\
\text {Стехіометрія } \\
\mathrm{Cu}(\mathrm{II}) \\
\left(O_{X}^{\times} C u_{i}^{\cdot *} V_{Z n}^{\prime \prime}\right)^{\times} \\
\end{array}$ & $I I^{\times}$ & $\begin{aligned} \lambda & =477 \\
\mathrm{E}_{\mathrm{A}} & =0.15 \\
\lambda & =508 \\
\mathrm{E}_{\mathrm{A}} & =0.24\end{aligned}$ & $\begin{array}{c}\lambda=355-370 \\
\mathrm{E}_{\mathrm{A}}=\sim 0.3 \\
\lambda=380 \\
\mathrm{E}_{\mathrm{A}}=0.37\end{array}$ \\
\hline $\begin{array}{c}\text { III } \\
\left(O_{X}^{\times} Z n_{i}^{* \cdot} V_{Z n}^{\prime}\right)^{\bullet} \\
\text { Надлишок халькогену } \\
\text { Cu(III) } \\
\left(O_{X}^{\times} C u_{i}^{* *} V_{Z n}^{\prime}\right)^{\bullet} \\
\end{array}$ & III $^{\bullet}$ & $\begin{aligned} \lambda & =735 \\
\mathrm{E}_{\mathrm{A}} & =0.87 \\
\lambda & =740 \\
\mathrm{E}_{\mathrm{A}} & =0.87\end{aligned}$ & $\begin{array}{c}\lambda=510 \\
\mathrm{E}_{\mathrm{A}}=1.1 \\
\lambda=520 \\
\mathrm{E}_{\mathrm{A}}=1.13\end{array}$ \\
\hline
\end{tabular}
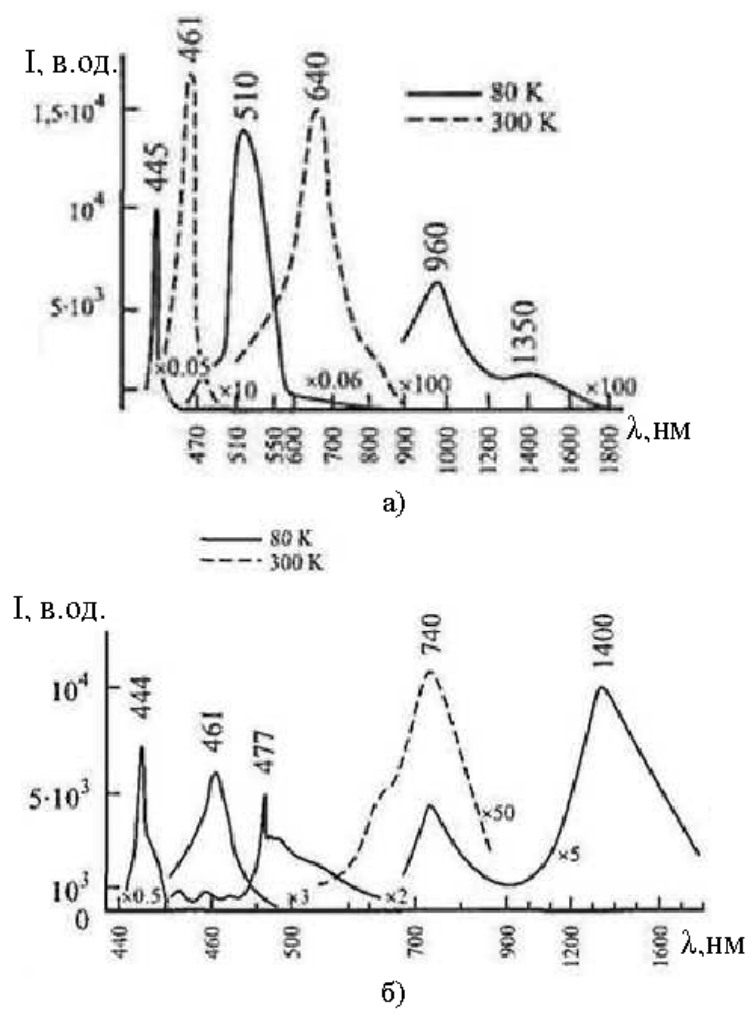

Рис. 2 Спектри катодолюмінесценції легованих міддю кристалів цинк селеніду із фоновою домішкою кисню при надлишку цинку (а) та халькогену (б) [3].

Аналогічно для $\mathrm{p}-\mathrm{Zn} X$ (надлишок халькогену у межах області гомогенності) легуючим кластером буде $V_{Z n}^{\prime \prime} V_{X}^{*}+X^{o} \rightarrow V_{Z n}^{\prime \prime} X_{X}^{*}$. Кристалоквазіхімічну формулу при врахуванні диспропорціювання ( $\mu$ ) вакансій цинку $V_{Z n}^{\prime \prime}$ можна представити як

$(1-\beta)\left(Z n_{Z n}^{\times} X_{X}^{\times}\right)+\beta\left(V_{Z n}^{\prime \prime} X_{X}^{*}\right) \rightarrow$

$\left(Z n_{1-\beta}^{\times} V_{\beta}^{\prime \prime}\right)_{Z n}\left(X_{1-\beta}^{\times} X_{\beta}^{*}\right)_{X} \rightarrow$

$\left(Z n_{1-\beta}^{\times} V_{\beta(1-\mu)}^{\prime \prime} V_{\beta \mu}^{\prime}\right)_{Z n} X_{X}^{\times}+2 \beta h+\beta \mu e^{\prime}$

де $\beta$ - відхилення від стехіометрії у сторону халькогену, $e^{\prime}$ i $h$ - електрони і дірки.

\section{Леговані кристали}

Маючи на увазі те, що процеси дефектоутворення у кристалах $\operatorname{Zn} X$ суттєво залежать від співвідношення кисню і міді [3], розглянемо два випадки. Зокрема, при переважанні кисню у легованих кристалах $\mathrm{Zn} X: \mathrm{Cu}$ будемо брати до уваги утворення комплексів як із цинком, так і 3 міддю (SA(I) - Cu(I), SAL(II) - Cu(II), III - Cu(III)), а міді - комплексів із міддю (Cu(I), $\mathrm{Cu}(\mathrm{II}), \mathrm{Cu}(\mathrm{III}))$ (Таблиця 1).

У першому випадку ([O] > [Cu]) будемо мати наступне.

Стехіометричні кристали. Для $\mathrm{ZnX}: \mathrm{O}$ легуючий кластер буде $V_{Z n}^{\prime \prime} V_{\ddot{X}}+\frac{1}{2} O_{2}^{0} \rightarrow V_{Z n}^{\prime} O_{\ddot{X}}$, а кристалоквазіхімічна формула

$$
\begin{aligned}
& (1-\varepsilon) Z n_{Z n}^{\times} X_{X}^{\times}+\varepsilon V_{Z n}^{\prime \prime} O_{X}^{*} \rightarrow \\
& \left(Z n_{1-\varepsilon}^{\times} V_{\varepsilon}^{\prime \prime}\right)_{Z n}\left(X_{1-\varepsilon}^{\times} O_{\varepsilon}^{\times}\right)_{X}+2 \varepsilon h
\end{aligned}
$$

Для легованих міддю кристалів $\mathrm{Zn} X: \mathrm{O}: \mathrm{Cu}-$ легуючий кластер $\quad V_{Z n}^{\prime \prime} V_{X}^{*}+C u^{o} \rightarrow C u_{Z n}^{\prime \prime} V_{X}^{*} \quad$ i кристалоквазіхімічна формула 


$$
\begin{aligned}
& (1-\sigma)\left\{\left(Z n_{1-\varepsilon}^{\times} V_{\varepsilon}^{\prime \prime}\right)_{Z n}\left(X_{1-\varepsilon}^{\times} O_{\varepsilon}^{\times}\right)_{X}+2 \varepsilon h \cdot+\sigma C u_{Z n}^{\prime \prime} V_{\ddot{X}} \rightarrow\right. \\
& \rightarrow\left(Z n_{1-2 \varepsilon(1-\sigma)}^{\times} V_{\varepsilon(1-\sigma)}^{\prime \prime}\right)_{Z n}\left(X_{(1-\sigma)(1-\varepsilon)}^{\times} V_{\ddot{\sigma}}\right)_{X}\left(O_{X}^{\times} C u_{i}^{\prime \prime} V_{Z n}^{\prime \prime}\right)_{\sigma}^{\times}\left(O_{X}^{\times} Z n_{i}^{*} V_{Z n}^{\prime \prime}\right)_{\varepsilon(1-\sigma)-\sigma}^{\times}+2 \sigma e+2 \varepsilon(1-\sigma) h .
\end{aligned}
$$

де $\varepsilon$ i $\sigma$ - частки легуючих кластерів із киснем і міддю.

Нестехіометричні кристали. У випадку нестехіометричних кристалів $\mathrm{n}-\mathrm{ZnX}: \mathrm{O}$ кристалоквазіхімічна формула буде мати вигляд

$$
\begin{aligned}
& \left.(1-x)\left[\left(Z n_{(1-\alpha)+\alpha \gamma}^{\times} V_{\alpha(1-\gamma)}^{\prime \prime}\right)\right)_{Z n}\left(X_{1-\alpha}^{\times} V_{\alpha}^{\ddot{\alpha}}\right)_{X}\left(Z n_{\alpha(1-\gamma)(1-\delta)} Z n_{\alpha(1-\gamma) \delta}\right)_{i}+\alpha(1+\gamma+\delta-\gamma \delta) e^{\prime}\right]+x\left(V_{Z n}^{\prime \prime} O_{\ddot{X}}\right) \rightarrow \\
& \rightarrow\left(Z n_{[(1-\alpha)+\alpha \gamma](1-x)}^{\times} V_{\alpha(1-\gamma)(1-x)+x}^{\prime \prime}\right)_{Z n}\left(X_{(1-\alpha)(1-x)}^{\times} V_{\dot{\alpha}(1-x)} O_{x}^{\times}\right)_{X}\left(Z n_{\alpha(1-\gamma)(1-\delta)(1-x)} Z n_{\alpha(1-\gamma)(1-x) \delta}\right)_{i}+ \\
& +\alpha(1-x)(1+\gamma+\delta-\gamma \delta) e^{\prime}+(\alpha(1-x)+2 x) h .
\end{aligned}
$$

Відповідно для кристалів $\mathrm{n}-\mathrm{Zn} X: \mathrm{O}: \mathrm{Cu}:$

$$
\begin{aligned}
& (1-z)\left\{\left(Z n_{(1-x)(1-\alpha+\alpha \gamma)}^{\times} V_{\alpha(1-\gamma)(1-x)+x}^{\prime \prime}\right)_{Z n}\left(X_{(1-\alpha)(1-x)}^{\times} V_{\dot{\alpha}(1-x)} O_{x}^{\times}\right)_{X}\left(Z n_{\alpha(1-\gamma)(1-\delta)(1-x)} Z n_{\alpha \delta(1-\gamma)(1-x)}\right)_{i}+\right. \\
& \left.\alpha(1-x)(1+\gamma+\delta-\gamma \delta) e^{\prime}+(\alpha(1-x)+2 x) h\right\}^{\prime \prime}+z C u_{Z n}^{\prime \prime} V_{\ddot{X}} \rightarrow \\
& \left(Z n_{(1-z)((1-x)(1-\alpha+\alpha \gamma)-x)+z}^{\times} V_{(1-z)(\alpha(1-\gamma)(1-x)+x)}^{\prime \prime}\right)_{Z n}\left(X_{(1-z)(1-\alpha)(1-x)}^{\times} V_{\dot{\alpha}(1-z)(1-x)+z}\right)_{X}\left(Z n_{\alpha(1-z)(1-\gamma)(1-\delta)(1-x)}^{\prime} Z n_{\alpha \delta(1-z)(1-\gamma)(1-x)}\right)_{i} \times \\
& \left(O_{X}^{\times} C u_{i}^{\prime} V_{Z n}^{\prime \prime}\right)_{z}^{\prime}\left(O_{X}^{\times} Z n_{i} V_{Z n}^{\prime \prime}\right)_{x(1-z)-z}^{\prime}+(\alpha(1-z)(1-x)(1+\gamma+\delta-\gamma \delta)+z) e^{\prime}+[(1-z)(\alpha(1-x)+3 x)] h
\end{aligned}
$$

де $\mathrm{x}$ i z - частки легуючих кластерів із киснем і міддю відповідно.

У випадку кристалів $\mathrm{p}-\mathrm{Zn} X: \mathrm{O}$ будемо мати аналогічно:

$\left.(1-y))\left(Z n_{1-\beta}^{\times} V_{\beta(1-\mu)}^{\prime \prime} V_{\beta \mu}^{\prime}\right)_{Z n} X_{X}^{\times}+2 \beta h+\beta \mu e^{\prime}\right]+y\left(V_{Z n}^{\prime \prime} O_{\ddot{X}}\right) \rightarrow$

$\left(Z n_{(1-y)(1-\beta)}^{\times} V_{\beta(1-y)(1-\mu)+y}^{\prime \prime} V_{\beta \mu(1-y)}^{\prime}\right)_{Z n}\left(X_{1-y}^{\times} O_{y}^{\times}\right)_{X}+(2 \beta(1-y)+2 y) h+\beta \mu(1-y) e^{\prime}$

Для $\mathrm{p}-\mathrm{Zn} X: \mathrm{O}: \mathrm{Cu}$ :

$(1-\lambda)\left\{\left(Z n_{(1-y)(1-\beta)}^{\times} V_{\beta(1-y)(1-\mu)+y}^{\prime \prime} V_{\beta \mu}^{\prime}(1-y)\right)_{Z n}\left(X_{1-y}^{\times} O_{y}^{\times}\right)_{X}+(2 \beta(1-y)+2 y) h+\right.$

$\left.\beta \mu(1-y) e^{\prime}\right\}+\lambda C u_{Z n}^{\prime \prime} V_{\ddot{X}} \rightarrow\left(Z n_{(1-\lambda)((1-y)(1-\beta)-y)+\lambda}^{\times} V_{(1-\lambda)(\beta(1-y)(1-\mu)+y)}^{\prime \prime} V_{\beta \mu}^{\prime}(1-\lambda)(1-y)\right)_{Z n} \times$

$\left(X_{(1-\lambda)(1-y)}^{\times} V_{\lambda}\right)_{X}\left(O_{X}^{\times} C u_{i}^{\prime} V_{Z n}^{\prime}\right)_{\lambda}^{\bullet}\left(O_{X}^{\times} Z n_{i}^{*} V_{Z n}^{\prime}\right)_{y(1-\lambda)-\lambda}^{\bullet}+$

$(1-\lambda)(2 \beta(1-y)+2 y) h+((1-\lambda)(\beta \mu(1-y)+y)+2 \lambda) e$

У другому випадку, за умови переважання вмісту міді $[\mathrm{Cu}]>[\mathrm{O}]$, при подвійному легуванні стехіометричних кристалів $\mathrm{ZnX}: \mathrm{O}: \mathrm{Cu}$ у кристалоквазіхімічній формулі, на відміну від (4), буде тільки комплекс із міддю :

$$
\begin{aligned}
& (1-\sigma)\left\{\left(Z n_{1-\varepsilon}^{\times} V_{\varepsilon}^{\prime \prime}\right)_{Z n}\left(X_{1-\varepsilon}^{\times} O_{\varepsilon}^{\times}\right)_{X}+2 \varepsilon h \cdot\right\}+\sigma C u_{z n}^{\prime \prime} V_{\ddot{X}} \rightarrow \\
& \left(Z n_{1-2 \varepsilon(1-\sigma)}^{\times} V_{\varepsilon(1-\sigma)}^{\prime \prime} C u_{\sigma-\varepsilon(1-\sigma)}^{\times}\right)_{Z n}\left(X_{(1-\sigma)(1-\varepsilon)}^{\times} V_{\ddot{\sigma}}\right)_{X}\left(O_{X}^{\times} C u_{i}^{\prime} V_{Z n}^{\prime \prime}\right)_{\varepsilon(1-\sigma)}^{\times}+(3 \sigma-\varepsilon(1-\sigma)) e^{\prime}+2 \varepsilon(1-\sigma) h .
\end{aligned}
$$

Відповідно для $\mathrm{n}-\mathrm{Zn} X: \mathrm{O}: \mathrm{Cu}:$

$$
\begin{aligned}
& (1-z)\left\{\left(Z n_{(1-x)(1-\alpha+\alpha \gamma)}^{\times} V_{\alpha(1-\gamma)(1-x)+x}^{\prime \prime}\right)_{Z n}\left(X_{(1-\alpha)(1-x)}^{\times} V_{\dot{\alpha}(1-x)} O_{x}^{\times}\right)_{X}\left(Z n_{\alpha(1-\gamma)(1-\sigma)(1-x)} Z n_{\alpha \delta(1-\gamma)(1-x)}\right)_{i}+\right. \\
& \left.\alpha(1-x)(1+\gamma+\delta-\gamma \delta) e^{\prime}+(\alpha(1-x)+2 x) h\right\}+z C u_{Z n}^{\prime \prime} V_{\ddot{X}} \rightarrow \\
& \left(Z n_{(1-z)(1-x)(1-\alpha+\alpha \gamma)}^{\times} C u_{z-x(1-z)}^{\times} V_{(1-z)(\alpha(1-\gamma)(1-x)+x)}\right)_{Z n}\left(X_{(1-z)}^{\times}(1-\alpha)(1-x)\right. \\
& \left(Z n_{\alpha(1-z)(1-\gamma)(1-\delta)(1-x)}^{\prime} Z n_{\alpha \delta(1-z)(1-\gamma)(1-x)}\right)_{i}\left(O_{X}^{\times} C u_{i}^{\prime} V_{Z n}^{\prime \prime}\right)_{X(1-z)}^{\prime} \times \\
& (\alpha(1-x)(1-z)(1+\gamma+\delta-\gamma \delta)+2 z-x(1-z)) e^{\prime}+[(1-z)(\alpha(1-x)+2 x)+z] h .
\end{aligned}
$$

та $\mathrm{p}-\mathrm{Zn} X: \mathrm{O}: \mathrm{Cu}$ :

$\left.(1-\lambda)\left\{\left(Z n_{(1-y)(1-\beta)}^{\times} V_{\beta(1-y)(1-\mu)+y}^{\prime \prime} V_{\beta \mu}^{\prime}(1-y)\right)\right)_{Z n}\left(X_{1-y}^{\times} O_{y}^{\times}\right)_{X}+(2 \beta(1-y)+2 y) h+\beta \mu(1-y) e^{\prime}\right\}+\lambda C u_{Z n}^{\prime \prime} V_{\ddot{X}} \rightarrow$

$\left(Z n_{(1-\lambda)(1-y)(1-\beta)}^{\times} C u_{\lambda-y(1-\lambda)}^{\times} V_{(1-\lambda)(\beta(1-y)(1-\mu)+y)}^{\prime \prime} V_{\beta \mu(1-\lambda)(1-y)}^{\prime}\right)_{Z n}\left(X_{(1-\lambda)(1-y)}^{\times} V_{\ddot{\lambda}}\right)_{X}\left(O_{X}^{\times} C u_{i}^{*} V_{Z n}^{\prime}\right)_{y(1-\lambda)}^{\bullet}+$

$+(1-\lambda)(2 \beta(1-y)+2 y) h+((1-\lambda)(\beta \mu(1-y)+y)+2 \lambda) e^{\prime}$ 


\section{Концентрація дефектів}

Запропоновані механізми дефектоутворення, а також кристалоквазіхімічні формули (1)-(11) дають аналітичну базу для визначення як концентрації окремих точкових дефектів та їхніх комплексів, так і холлівської концентрації носіїв струму $\mathrm{n}_{\mathrm{H}}$ залежно від вмісту легуючих домішок $\mathrm{O}$ i $\mathrm{Cu}$, a також величини відхилення від стехіометричного складу $(\alpha, \beta)$ у базовій матриці $\mathrm{ZnX}$. Так, зокрема, у випадку кристалів n$\mathrm{Zn} X: \mathrm{O}: \mathrm{Cu}$ згідно $(6,10)$ отримаємо:

$\left\lfloor V_{Z n}^{\prime \prime}\right\rfloor=A(1-z)(\alpha(1-\gamma)(1-x)+x)$,

$\left\lfloor V_{X}^{\bullet}\right\rfloor=A(\alpha(1-z)(1-x)+z)$,

$\left[Z n_{i}^{\bullet}\right\rfloor=A \alpha(1-z)(1-\gamma)(1-\delta)(1-x)$,

$\left[Z n_{i}^{\bullet \bullet}\right\rfloor=A \alpha \delta(1-z)(1-\gamma)(1-x)$.

При $[\mathrm{O}]>[\mathrm{Cu}]$ згідно (6)

$\left[\left(O_{X}^{\times} C u_{i}^{\bullet} V_{Z n}^{\prime \prime}\right)^{\prime}\right]=A z$,

$\left[\left(O_{X}^{\times} Z n_{i}^{\bullet} V_{Z n}^{\prime \prime}\right)^{\prime}\right]=A(x(1-z)-z)$,

$n=A(\alpha(1-z)(1-x)(1+\gamma+\delta-\gamma \delta)+z)$,

$p=A[(1-z)(\alpha(1-x)+3 x)]$.

При $[\mathrm{Cu}]>[\mathrm{O}]$ згідно (10)

$\left[\left(O_{X}^{\times} C u_{i}^{\bullet} V_{Z n}^{\prime \prime}\right)^{\prime}\right]=A x(1-z)$,

$n=A(\alpha(1-z)(1-x)(1+\gamma+\delta-\gamma \delta)+2 z-x(1-z))$,

$p=A[(1-z)(\alpha(1-x)+2 x)+z]$.

Рівняння електронейтральності та холлівська концентрація для $[\mathrm{O}]>[\mathrm{Cu}]$ згідно (6)

$2\left[V_{Z n}^{\prime \prime}\right]+\left[\left(o_{X}^{\times} C u_{i}^{\bullet} V_{Z n}^{\prime \prime}\right)^{\prime}\right]+\left[\left(o_{X}^{\times} Z n_{i}^{\bullet} V_{Z n}^{\prime \prime}\right)^{\prime}\right]+n=$, $\left[V_{X}^{\bullet}\right]+\left[Z n_{i}^{\bullet}\right]+2\left[Z n_{i}^{\bullet \bullet}\right]+p$

$n_{H}=|n-p|=\mid\left[V_{X}^{\bullet}\right]+\left[Z n_{i}^{\bullet}\right]+2\left[Z n_{i}^{\bullet \bullet}\right]-2\left[V_{Z n}^{\prime \prime}\right]-$

$\left[\left(o_{X}^{\times} C u_{i}^{\bullet} V_{Z n}^{\prime \prime}\right)^{\prime}\right]-\left[\left(o_{X}^{\times} Z n_{i}^{\bullet} V_{Z n}^{\prime \prime}\right)^{\prime}\right] \mid$

і для $[\mathrm{Cu}]>[\mathrm{O}]$ згідно (10)

$2\left[V_{Z n}^{\prime}\right]+\left[\left(O_{X}^{\times} C u_{i}^{\bullet} V_{Z n}^{\prime}\right)^{\prime}\right]+n=$

$\left[V_{X}^{\bullet}\right]+\left[Z n_{i}^{\bullet}\right]+2\left[Z n_{i}^{\bullet \bullet}\right]+p$

$n_{H}=|n-p|=\mid\left[V_{X}^{\bullet}\right]+\left[Z n_{i}^{\bullet}\right]+2\left[Z n_{i}^{\bullet \bullet}\right]-$

$2\left[V_{Z n}^{\prime \prime}\right]-\left[\left(O_{X}^{\times} C u_{i}^{\bullet} V_{Z n}^{\prime \prime}\right)^{\prime}\right]$

де $A=\frac{2 Z}{a^{3}}(Z=4-$ кількість структурних одиниць в елементарній комірці, $a$ - параметр елементарної комірки сфалеритної структури).

Аналогічні вирази можна отримати і для інших умов легування.

\section{Обговорення результатів}

Отримані кристалоквазіхімічні формули (1)-(11) та приведені результати розрахунків (Рис. 3, 4) вказують на наступне. У випадку стехіометричного складу $\mathrm{ZnX}$ (Рис. 3a) домінуючими точковими дефектами $є$ негативно заряджені двозарядні вакансії цинку $V_{Z n}^{\prime \prime}$ та позитивні однозарядні вакансії халькогену $V_{X}^{\bullet}$. Iз збільшенням концентрації міді, $[\mathrm{Cu}]>0,8 \cdot 10^{-5}$ ат. частки, при незначній зміні концентрації $V_{Z n}^{\prime \prime}$ має місце зростання кількості вакансій халькогену (Рис. 3а - крива 2). Це зумовлює значне зростання концентрації електронів (Рис. 3а - крива 10) і відбувається конверсія провідності 3 р- на n-тип. Подальше збільшення частки міді призводить до зростання холлівської концентрації $\mathrm{n}_{\mathrm{H}}$ за рахунок збільшення $\left[V_{X}^{\bullet}\right]$, що перевищують $\left[V_{Z n}^{\prime \prime}\right]$ (Рис. 3a криві 1, 2).

Для нестехіометричних кристалів $\mathrm{n}-\mathrm{Zn} X: \mathrm{O}: \mathrm{Cu}$ переважають такі точкові дефекти: $V_{X}^{\bullet}, V_{Z n}^{\prime \prime}$ та $Z n_{i}^{\bullet}$. Концентрація двозарядних міжвузлових атомів цинку [ $Z n_{i}^{\bullet \bullet}$ на декілька порядків нижча. Що стосується комплексів, то із збільшенням вмісту міді має місце зростання $\mathrm{Cu}(\mathrm{I})$ та зменшення SA(I) (Рис. 3б, Таблиця 1). При незначному вмісті міді (менше $0,3 \cdot 10^{-4}$ ат. частки) кристали володіють р-типом провідності, за який відповідальними $є$ двозарядні вакансії цинку (Рис. $3 б$ - крива 1). При збереженні діркової провідності холлівська концентрація дещо зменшується із збільшенням вмісту міді за рахунок зростання концентрації [ $\left.V_{X}^{\bullet}\right]$ та $\left[Z n_{i}^{\bullet}\right]$, парціальний коефіцієнт компенсації яких зростає. Внесок інших дефектів $є$ менш суттєвим, на що, зокрема, вказує значення їхніх коефіцієнтів компенсації. При подальшому збільшенні вмісту міді концентрація дірок зменшується, відбувається конверсія провідності матеріалу 3 p- на n-тип i подальше зростання концентрації електронів (рис. 36 - криві 9, 10). Це зумовлено зростанням концентрації вакансій халькогену та цинку у міжвузлях, парціальний коефіцієнт компенсації яких зростає.

При відхиленні від стехіометрії у сторону халькогену у $\mathrm{p}-\mathrm{Zn} X: \mathrm{O}: \mathrm{Cu}$ переважаючими точковими дефектами $€ V_{Z n}^{\prime \prime}$ та $V_{X}^{\bullet}$ (Рис. 3в - криві $1,2)$. Концентрація однозарядних вакансій цинку незначна. Аналогічно як і у випадку $\mathrm{n}-\mathrm{Zn} X: \mathrm{O}: \mathrm{Cu}$ $([\mathrm{O}]>[\mathrm{Cu}])$, при збільшенні концентрації міді спостерігається зменшення SA(II) та зростання $\mathrm{Cu}(\mathrm{II})$ (Рис. 3в, Таблиця 1). При незначному вмісті міді (менше $0,6 \cdot 10^{-4}$ ат. частки), зростає концентрація вакансій халькогену, однак вакансії цинку мають домінуючий вплив (Рис. 3в - крива 1). При цьому, із збільшенням ат. частки міді концентрація вакансій цинку зменшується, а 


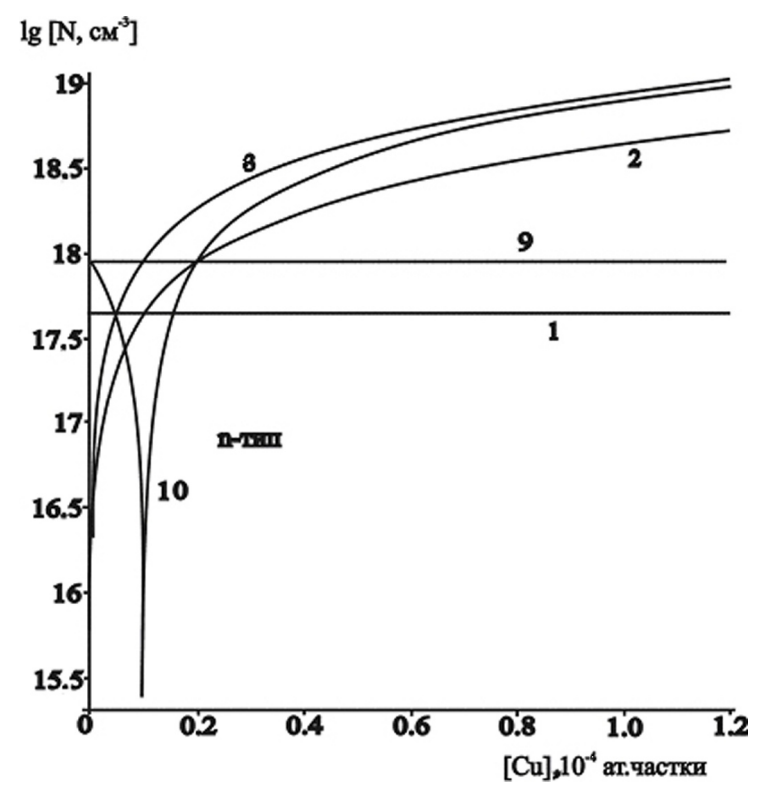

$\mathrm{a}$

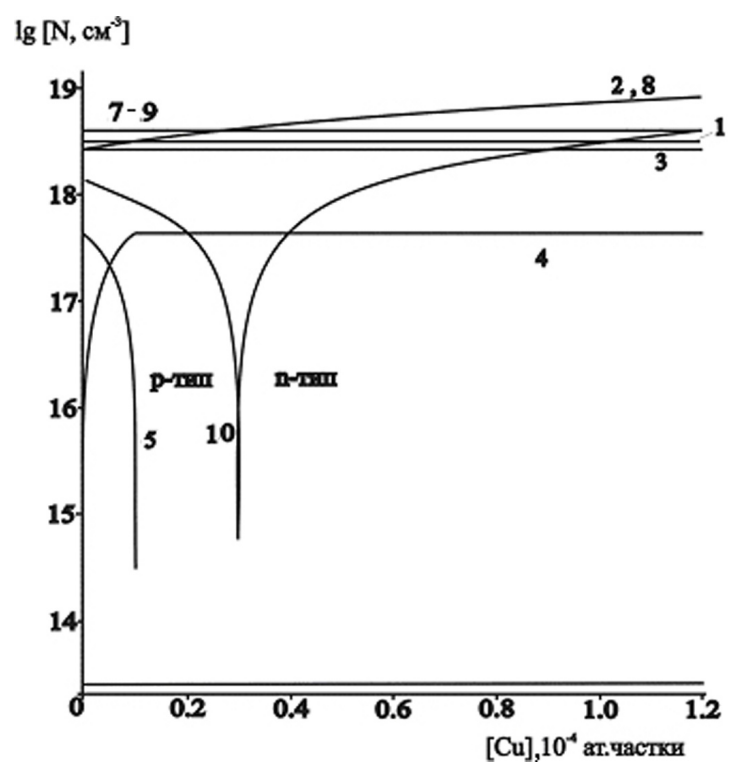

6

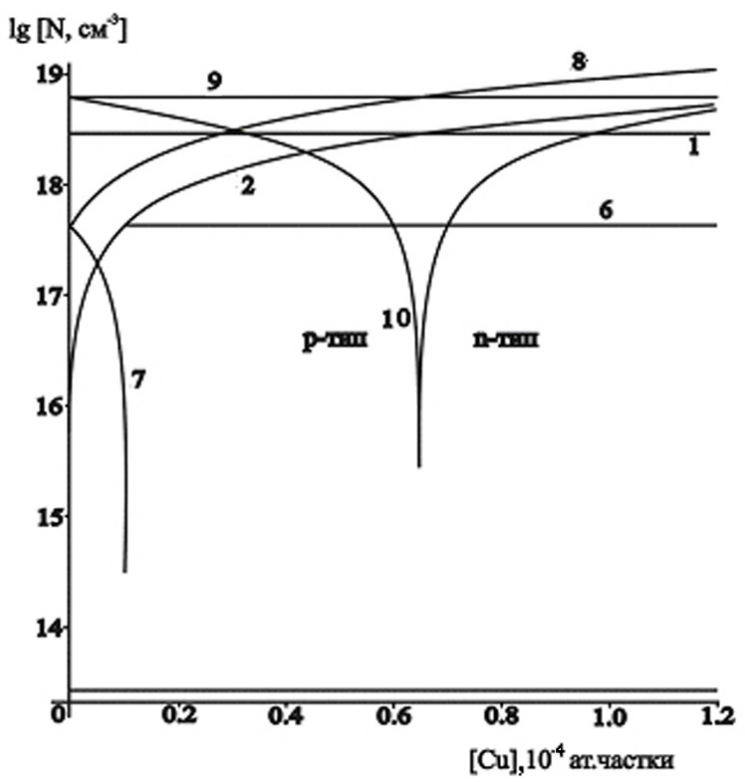

Рис. 3 Залежність концентрації переважаючих точкових дефектів $1-V_{Z n}^{\prime \prime}, 2-V_{X}^{\cdot}, 3-Z n_{i}$, комплексів $4-\left(o_{X}^{\times} C u_{i}^{\prime} V_{Z n}^{\prime \prime}\right)^{\prime}, 5-\left(o_{X}^{\times} Z n_{i}^{\prime} V_{Z n}^{\prime \prime}\right)^{\prime}, 6-\left(o_{X}^{\times} C u_{i}^{\cdot} V_{Z n}^{\prime}\right)^{\bullet}, 7-\left(o_{X}^{\times} Z n_{i}^{*} V_{Z n}^{\prime}\right)^{\bullet}$, основних носіїв $8-\mathrm{n}$ і $9-\mathrm{p}$ та холлівської концентрації $10-\mathrm{n}_{\mathrm{H}}$ у кристалах: a $-\mathrm{Zn} X: \mathrm{O}: \mathrm{Cu}$ (при вмісті кисню $\varepsilon=10^{-5}$ ат. частки); б $\mathrm{n}-\mathrm{Zn} X: \mathrm{O}: \mathrm{Cu}\left(\alpha=6 \cdot 10^{-5}\right.$ ат. частки, $\delta=10^{-5}, x=10^{-5}$ ат. частки, $\left.\gamma=10^{-5}\right)$; в $-\mathrm{p}-\mathrm{Zn} X: \mathrm{O}: \mathrm{Cu}\left(\beta=6 \cdot 10^{-5}\right.$ ат. частки, $\mu=10^{-5}, y=10^{-5}$ ат. частки) від вмісту легуючої домішки $[\mathrm{Cu}]$.

концентрація вакансій халькогену зростає. Це зумовлює певне зменшення холлівської концентрації при збереженні р-типу провідності (Рис. 3в - крива 10). При збільшенні вмісту міді відбувається конверсія провідності 3 p- на n-тип, оскільки концентрація $\left[V_{X}^{\bullet}\right]$ значно зростає.

Для $\mathrm{n}-\mathrm{Zn} X: \mathrm{O}: \mathrm{Cu}$ збільшення відхилення від стехіометрії у сторону цинку не впливає на положення p-n-переходу (Рис. 4a). При збільшенні відхилення від стехіометрії у сторону халькогену у
p-ZnX:O:Cu p-n-перехід реалізується при більшій концентрації міді (Рис. 4б).

\section{Висновки}

1. Запропоновано кристалоквазіхімічні формули легованих кристалів $\mathrm{Zn} X: \mathrm{O}: \mathrm{Cu}$ стехіометричного складу та n- i p-типу провідності.

2. Показано, що за умови $[\mathrm{O}]>[\mathrm{Cu}]$ для n-типу провідності переважають $V_{Z n}^{\prime \prime}, \quad V_{X}^{\bullet}, Z n_{i}^{\bullet}$ та 


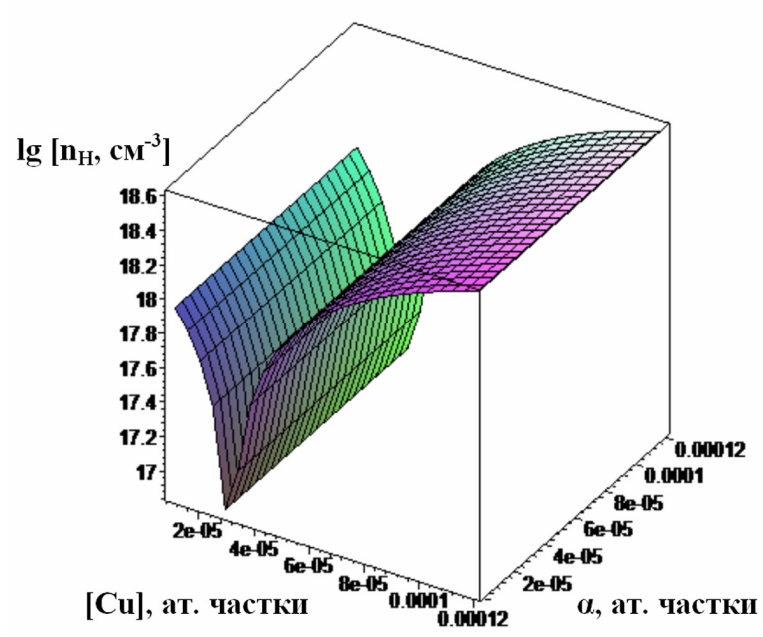

a

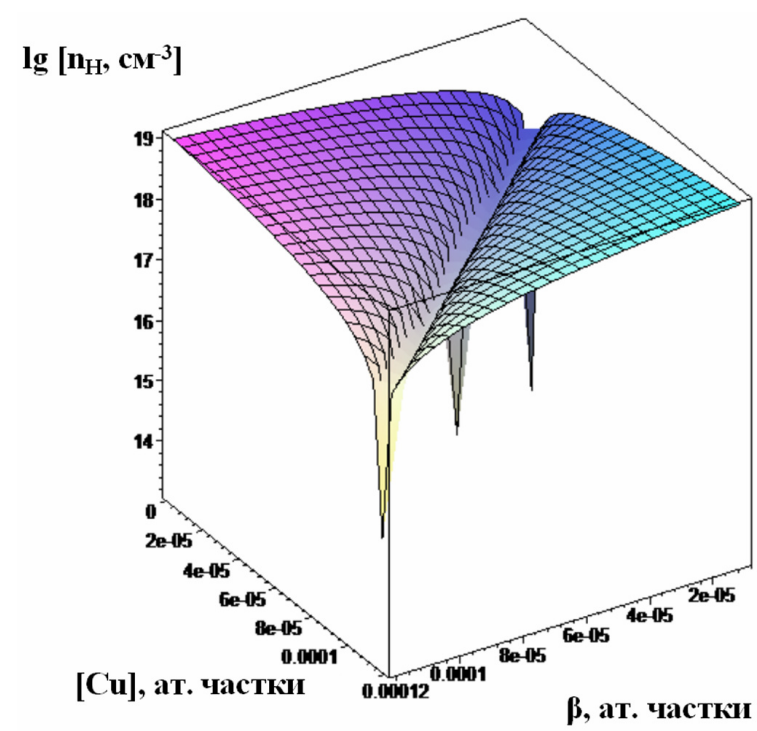

б

Рис. 4 Просторові залежності холлівської концентрації $\mathrm{n}_{\mathrm{H}}$ для кристалів n-ZnX:O:Cu (a) та p$\mathrm{ZnX:O:Cu} \mathrm{(б)} \mathrm{від} \mathrm{відхилення} \mathrm{від} \mathrm{стехіометрії} \alpha(\beta)$ та вмісту легуючої домішки $[\mathrm{Cu}]$.

комплекси $\left(O_{X}^{\times} Z n_{i}^{\bullet} V_{Z n}^{\prime \prime}\right)^{\prime},\left(O_{X}^{\times} C u_{i}^{\bullet} V_{Z n}^{\prime \prime}\right)^{\prime}$. Для р-типу провідності переважають $V_{Z n}^{\prime \prime}$ та комплекси $\left(O_{X}^{\times} Z n_{i}^{\bullet \bullet} V_{Z n}^{\prime}\right)^{\bullet},\left(O_{X}^{\times} C u_{i}^{\bullet \bullet} V_{Z n}^{\prime}\right)^{\bullet}$.

3. За умови $[\mathrm{Cu}]>[\mathrm{O}]$ для п-типу провідності переважаючими дефектами $€ V_{Z n}^{\prime \prime}, V_{X}^{\bullet}, Z n_{i}^{\bullet}$, а комплексом - $\left(O_{X}^{\times} C u_{i} V_{Z n}^{\prime \prime}\right)^{\prime}$. Для р-типу провідності $-V_{Z n}^{\prime \prime}, V_{s e}^{\bullet}$ та комплекс $\left(O_{X}^{\times} C u_{i}^{*} V_{Z n}^{\prime}\right)^{\bullet}$.

\section{Подяка}

Робота виконується в рамках гранту Міністерства освіти i науки України (реєстраційний номер 0107U006768).

\section{Літературні посилання}

[1] А.Н. Георгобиани, М.Б. Котляревский, Изв. АН СССР, Сер. Физ. 49(10) (1985) 1916-1922.

[2] В.М. Бабич, Н.И. Блукан, Е.Ф. Венгер, Кислород в монокристаллах кремния, Интерпресс ЛТД, Киев, 1997, 240 с.

[3] Н.К. Морозова, И.А. Каретников, Физ. Техн. Полупровод. 35(1) (2001) 25-33.

[4] Л.Й. Межиловська, Г.Я. Бабущак, П.В. Жуковскі, Фіз. Хім. Тверд. Тіла 7(3) (2006) 618-623. 